\section{Regulation of NKG2D Expression and Signaling by Endocytosis}

Rosa Molfetta, ${ }^{1}$ Linda Quatrini, ${ }^{1,4}$ Beatrice Zitti, ${ }^{1}$ Cristina Capuano, ${ }^{2}$ Ricciarda Galandrini, ${ }^{2}$ Angela Santoni, ${ }^{1,3, \star}$ and Rossella Paolini ${ }^{1, \star}$

NKG2D is an activating receptor that can bind to a large number of stressinduced ligands that are expressed in the context of cancer or viral infection. This receptor is expressed on many cytotoxic lymphocytes, and plays a crucial role in antitumor and antiviral immune responses. However, exposure to NKG2D ligand-expressing target cells promotes receptor endocytosis, ultimately leading to lysosomal receptor degradation and impairment of NKG2D-mediated functions. Interestingly, before being degraded, internalized receptors can signal from the endosomal compartment, leading to the appropriate activation of cellular functional programs. This review summarizes recent findings on ligand-induced receptor internalization, with particular emphasis on the role of endocytosis in the control of both NKG2D-mediated intracellular signaling and receptor degradation.

\section{NKG2D and Sensing of Cellular Stress}

The natural killer (NK) receptor group 2, member D (NKG2D) is one of the best-characterized NK cell-activating receptors and is also expressed on human $\mathrm{CD}^{+} \alpha \beta T$ cells and murine activated $\mathrm{CD}^{+} \alpha \beta$ T cells, human activated $\mathrm{CD} 4^{+} \alpha \beta$ T cells, and $\gamma \delta \mathrm{T}$ cells. NKG2D is a fundamental player in antitumor and antiviral immune responses: it recognizes ligands absent or poorly expressed on normal cells that have been upregulated upon cellular stress, microbial infection, malignant transformation, or autoimmunity [1-4].

The prominent role of NKG2D in tumor immune surveillance was first demonstrated in murine models by ectopic expression of NKG2D ligands, and this was found to be sufficient to cause tumor rejection in syngeneic mice [5,6]. Moreover, NKG2D neutralization by means of specific antibodies significantly impaired host protection from de novo tumorigenesis [7]. Remarkably, NKG2D-deficient mice also showed enhanced susceptibility to the development of tumors [8] and cytomegalovirus infection [9], thus confirming the role of NKG2D-mediated immune surveillance.

Accumulating evidence demonstrate that persistent exposure to NKG2D ligand-expressing target cells promotes receptor downmodulation, with consequent impairment of NKG2Dmediated functions [10-15]. Upon ligand binding, NKG2D is subjected to internalization and lysosomal degradation, and these processes require the ubiquitin pathway $[16,17]$. Nevertheless, recent evidence supports a role for NKG2D ubiquitin-dependent endocytosis not only in the clearance of receptor from the cell surface but also in the propagation of intracellular signals [17].

\section{Trends}

The NKG2D receptor on cytotoxic lymphocytes recognizes ligands upregulated during viral infection and tumor transformation, allowing the killing of stressed cells.

A distinctive feature of the NKG2D receptor resides in its interaction with a large number of MHC-I related ligands.

Persistent exposure to NKG2D ligands causes receptor endocytosis, rendering cytotoxic lymphocytes hyporesponsive to NKG2D ligand-bearing target cells.

NKG2D ligands are not all equivalent in their ability to induce receptor endocytosis, resulting in different functional outcomes.

NKG2D endocytosis is not only instrumental for the clearance of activated receptors from the cell surface but can also elicit functional responses controlling NKG2D-mediated signal propagation.

${ }^{1}$ Department of Molecular Medicine Institute Pasteur-Fondazione Cenci Bolognetti, 'Sapienza' University of Rome, 00161, Rome, Italy

${ }^{2}$ Department of Experimental Medicine, 'Sapienza' University of Rome, 00161 Rome, Italy

${ }^{3}$ Istituto Di Ricovero e Cura a Carattere Scientifico (IRCCS), Neuromed, Pozzilli, IS, Italy ${ }^{4}$ Present address: Centre d'Immunologie de Marseille-Luminy, Parc Scientifique et Technologique de 
This review summarizes the molecular mechanisms involved in ligand-induced NKG2D downmodulation, focusing mainly on receptor endocytosis. The role of human NKG2D ubiquitindependent endocytosis in the control of signal propagation and NK cell function is also discussed.

\section{NKG2D Receptor Structure and Signaling Pathways}

NKG2D is a C-type lectin receptor: human NKG2D and the long splice-variant form of mouse NKG2D (NKG2D-L) assemble as hexameric complexes composed of two NKG2D molecules associated with four molecules of the signaling adapter DNAX-associated protein 10 (DAP10) [18], which displays a cytoplasmic YINM motif and recruits the regulatory subunit of phosphoinositide-3 kinase (PI3K), p85, that is responsible for stimulatory signals [19,20] (Figure 1A). In mice, activated NK cells express an additional shorter NKG2D isoform (NKG2D-S) that can associate with either DAP10 or DAP12, the latter containing an immune tyrosine-based activation motif (ITAM) capable of binding to Syk family protein tyrosine kinases [21,22] (Figure 1A).

Upon ligand binding, DAP10 is phosphorylated in its YINM cytoplasmic motif by Src family kinases [23], but the contribution of other kinases has not been excluded. In this regard, Horng and colleagues found that 'priming' of NKG2D-mediated signaling requires IL-15-activated JAK3 that is responsible for maximal DAP10 phosphorylation [24]. The phosphorylated YINM motif of DAP10 can directly associate with the adaptor protein Grb2 and PI3K subunit p85 $[20,23,25,26]$. PI3K leads to activation of the kinase Akt and downstream survival pathways, while Grb2 directly recruits the guanine nucleotide exchange factor Vav1, and this binding is both necessary and sufficient for the tyrosine phosphorylation of Vav1, phospholipase $\mathrm{C} \gamma$ (PLC 2 2), and of Src homology 2 (SH2) domain-containing leukocyte protein of $76 \mathrm{kDa}$ (SLP-76).

Engagement of human NKG2D potentiates T cell receptor (TCR)-induced cytotoxic function in $\mathrm{CD}^{+}$T cells [19], and NKG2D engagement alone on freshly isolated human NK cells is not sufficient to promote their full functional response [27]. Indeed, NK cell activation depends on a balance of activating and inhibitory signals, the latter being delivered by receptors for $\mathrm{MHC}$ class I molecules. The full activation of naive NK cells requires the synergistic co-engagement of at least two different activating receptors to overcome the inhibitory signal. In the context of NK cell functional responses mediated by NKG2D, its co-engagement with 2B4 or NKp46 has been reported to be required [27].

In activated murine NK cells, association with the DAP12 adapter enables NKG2D-mediated activation of Syk and ZAP-70 tyrosine kinases which, upon phosphorylation of the adaptor protein LAT, promote the recruitment and phosphorylation of PLC $\gamma$ and the activation of PI3K. Both these events are required for the exocytosis of Iytic granules and the activation of the transcriptional program leading to cytokine production $[21,22]$.

\section{NKG2D Ligand Heterogeneity and Expression}

A distinctive feature of NKG2D receptor resides in its interaction with a large number of MHC-I related ligands that exhibit considerable allelic variation [1-4]. The existence of various NKG2D ligands may be explained by a wide range of stress pathways that upregulate their expression in different cell types, thus enabling the same receptor to be activated in diverse contexts, as well as to increase the efficiency of antiviral and anticancer response. Moreover, the selection pressures exerted on the immune system by pathogen infections or by mechanisms of cancer immunoediting may have also favored the appearance of different ligands [1,2].

Two families of ligands for the human NKG2D receptor have been described: the highly polymorphic 'MHC class I related proteins', MICA and MICB, and the 'UL16 binding proteins' ULBPs (also known as the retinoic acid early transcripts, RAETs) [2-4] (Figure 1B). Similarly to
Luminy Case 906, 13288 Marseille CEDEX 09, France.

${ }^{*}$ Correspondence: angela.santoni@uniroma1.it (A. Santoni) and rossella.paolini@uniroma1.it (R. Paolini). 
classical MHC-I molecules, MICA/B proteins contain $\propto 1, \propto 2$, and $\propto 3$ domains; however, they do not associate with $\beta 2$-microglobulin and do not present antigenic peptides. Six ULBP molecules (ULBP1-6) have been identified to date. They lack the $\propto 3$ domain and differ in membrane anchoring: ULBP4 and 5 are transmembrane (TM) proteins while ULBP1-3 and 6 are linked to plasma membrane by a glycosylphosphatidylinositol (GPI) anchor (see Glossary). However, this classification is not straightforward because ULBP2 and 5 are expressed either as GPI or TM proteins [28,29]. Similarly, the most frequent MICA allele, namely MICA*008, contains a frameshift mutation before the TM region that leads to an early stop codon, resulting in the expression of a truncated GPI-linked protein [30].

Orthologs of the human ULPB/RAET1 family are the only ligands present in mice, and these comprise three subgroups of proteins: the GPI-linked RAE-1 (retinoic acid early inducible-1) ligands (five different isoforms); MULT1 (murine UL16-binding protein-like transcript 1) TM protein; and H60 ligands including one GPI-linked and two TM isoforms [2,3] (Figure 1B).

Although MICA is constitutively expressed on the gastrointestinal epithelium [31], and mRNA for both MICA/B and ULBP proteins can be found in some normal cells [32], the cell-surface levels of NKG2D ligands are either absent or low on the vast majority of healthy cells. Instead, NKG2D ligand expression is tightly regulated at the transcriptional, post-transcriptional, and posttranslational levels by different physiological and pathological 'stress' conditions, including mitosis, viral infection, and cancer, through the activation of different signaling pathways. Among these, the induction of NKG2D ligands by the DNA damage response (DDR) pathway has been well documented [2,33-35] (Box 1).

Moreover, autoimmune diseases such as rheumatoid arthritis and celiac disease are associated with increased expression of MICA on synovial tissue or intestinal epithelium, respectively, that may contribute to the activation of autoreactive NKG2D-expressing lymphocytes [36-38].

To counteract NKG2D ligand upregulation, several viruses (e.g., HCMV, HIV-1) have developed the ability to inhibit cell-surface ligand expression and, as a consequence, to decrease the susceptibility of infected cells to NK cell-mediated cytotoxicity [39]. Cancer cells have also developed multiple mechanisms to escape from NKG2D-mediated immune responses. One of these mechanisms depends on antitumor pressure exerted by cytotoxic lymphocytes that favors the selection of tumor cell clones able to release soluble NKG2D ligands from their plasma membrane through alternative splicing, proteolytic shedding, or exosome secretion. Alternative processing of ULBP4 and 5 transcripts generate a secreted ligand form that can inhibit target cell recognition in vitro [40,41]. Different TM allelic variants of MICA, MICB [42-44], and ULBP2 [15] are shed upon proteolytic cleavage mediated by ADAM (a disintegrin and metalloproteinase) family metalloproteases, whereas the allelic variant MICA*008 and ULBP3, both expressed on the plasma membrane as GPI-linked molecules, are released in exosomes $[45,46]$.

\section{Regulation of NKG2D Expression and Functional Consequences}

NKG2D expression is positively or negatively regulated by different cytokines, including IL-2, IL-7, IL-12, IL-15, and type I interferons (IFNs), which have been shown to increase NKG2D surface expression, whereas IL-21, IFN- $\gamma$, and TGF- $\beta$ have the opposite effect $[4,47]$. However, NKG2D expression is mainly regulated upon exposure to membrane-bound or soluble NKG2D ligands [10-15,48,49] (Figure 2).

The first evidence of NKG2D downmodulation stems from studies on $\mathrm{CD}^{+}$tumor-infiltrating $\mathrm{T}$ lymphocytes and peripheral blood mononuclear cells (PBMCs) isolated from patients with different MICA/B-positive epithelial tumors [10]. Groh and coworkers showed that NKG2D

\section{Glossary}

ADAM (a disintegrin and metalloproteinase) family: a group of transmembrane (TM) and secreted metalloproteases, also known as sheddases, that are able to cut off or shed the extracellular portions of TM proteins.

Clathrin-mediated endocytosis: the uptake of material into eukaryotic cells from the surface using clathrincoated vesicles. It is fundamental to neurotransmission, signal transduction, and the regulation of many plasma-membrane activities.

\section{DNA damage response (DDR): a} network of interactive pathways that sense, signal, and repair different types of DNA damage. The response initially arrests cell-cycle progression, allowing time to repair the DNA lesions, but may also induce transcriptional programs, enhance DNA repair pathways, and, when the level of damage is severe, promote apoptosis.

E3 ubiquitin ligase: an enzyme that recognizes a protein substrate and catalyzes the transfer of ubiquitin to that substrate. Ubiquitination by E3 ligases regulates several cellular processes including DNA repair, endocytosis, protein trafficking, and the cell cycle.

Exosomes: specialized nano-sized vesicles of endocytic origin that are released by many cell types and might act as regulators of intercellular communication.

Glycosylphosphatidylinositol (GPI) anchor: a glycolipid structure that is added post-translationally to the Cterminus of many eukaryotic proteins, and that anchors the modified protein in the outer plasma-membrane leaflet.

\section{Immune synapse (or} immunological synapse): a stable contact zone between an immune cell and another cell at which molecules accumulate, by analogy to neuronal synapses.

Trogocytosis: the transfer of cellsurface proteins and membrane patches from one cell to another through cell-cell contact. 
(A)
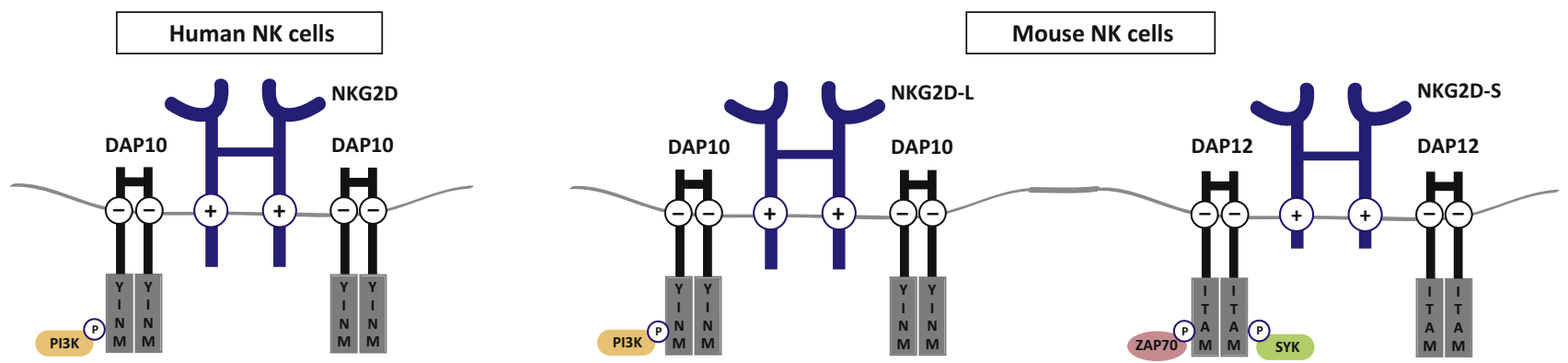

(B)

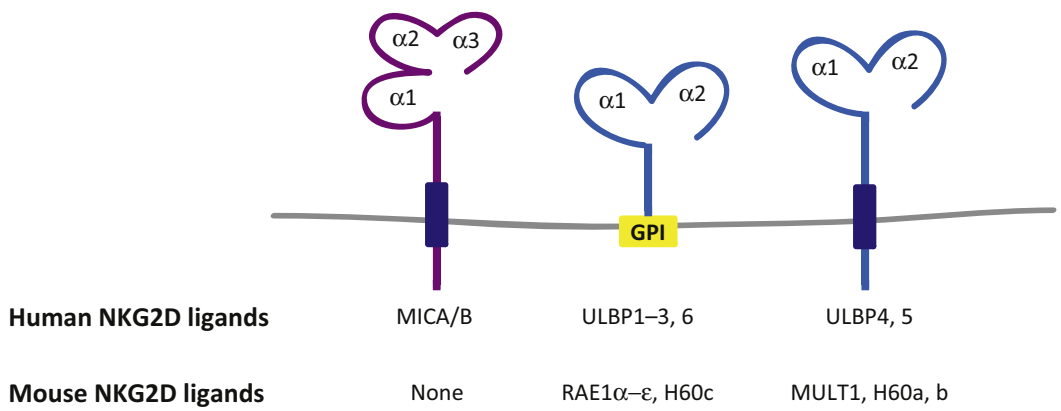

Trends in Immunology

Figure 1. NKG2D Receptor Complex and Its Ligands. (A) Schematic representation of NKG2D receptor complexes on human (left) and murine (right) NK cells. Human NKG2D and the long splice-variant form of mouse NKG2D (NKG2D-L) assemble as hexameric complexes composed of two NKG2D molecules associated with four molecules of DAP10. In mice, activated NK cells express an additional shorter NKG2D isoform (NKG2D-S) that can associate with either DAP10 or DAP12. DAP10 and DAP12 signaling motifs and the main kinases involved in signal propagation are depicted. (B) Schematic representation of human and mouse NKG2D ligands. Extracellular domains and the membrane anchor mode of the different ligands are indicated. Abbreviations: DAP, DNAX-associated protein; ITAM, immunoreceptor tyrosine-based activation motif; MIC, MHC class I related protein; NK cell; natural killer cell; PI3K, phosphoinositide-3 kinase; RAE, retinoic acid early-inducible; ULBP, UL16 binding protein.

surface expression on $\mathrm{CD}^{+} \mathrm{T}$ lymphocytes is reduced upon direct contact with MIC-expressing target cells, as well as by chronic stimulation with sera containing soluble MICA/B, leading to impairment of NKG2D ability to costimulate $\mathrm{CD} 8^{+} T$ cell effector functions. These results suggest that NKG2D ligands can modulate cell responsiveness and promote immune evasion. These findings were also confirmed in vivo. Indeed, in transgenic mice overexpressing MICA, prolonged exposure to this cell-bound ligand led to systemic NKG2D downmodulation. As a consequence, both NK and $\mathrm{CD}^{+} \mathrm{T}$ cells failed to reject MICA-positive tumors and showed defective immune surveillance of intracellular pathogens [13].

The ability to downmodulate NKG2D expression was also reported for ULBP ligands. In particular, reduced NKG2D expression was observed on NK cells exposed to ULBP2-transfected cells or primary ULBP2 ${ }^{+}$leukemic cells [15].

By comparing the ability of membrane-bound MICA and ULBP2 to induce NKG2D downmodulation in human NK cells, we found that both ligands reduce cell-surface receptor expression [16]. However, MICA promotes stronger downmodulation than ULBP2, leading to a more severe impairment of NKG2D-dependent NK cell cytotoxicity. The differential abilities of MICA versus ULBP2 to induce receptor downregulation may be attributable to their distinct 


\section{Box 1. NKG2D Ligand Regulation by the DDR}

A major signaling pathway implicated in the upregulation of NKG2D ligand expression consists of the activation of the DDR initiated by three members of the phosphatidylinositol 3-kinase-like serine/threonine protein family: ataxia telangiectasia mutated (ATM), ATM- and Rad3-related (ATR), and the DNA-dependent protein kinase (DNA-PK) [2,33-35,61]. These kinases and their downstream mediators, including the checkpoint kinases CHK1 and CHK2, are activated by DNA damage sensor proteins and promote cell-cycle arrest and DNA repair, or, if the damage is not successfully repaired, program the cell to undergo senescence or apoptosis.

Because ATM/ATR regulate cell division, their activation during cellular proliferation could represent a signal resulting in NKG2D ligand expression on healthy cells. Indeed, several studies have reported a correlation between NKG2D ligand expression and cell proliferation [31,62], and identified the involvement of NF- $\mathrm{KB}$ and the E2F family of transcription factors $[62,63]$.

Infection by several viruses, including herpesvirus, adenoviruses, and retroviruses, activates the DDR-mediated pathways and concurrently promotes upregulation of NKG2D ligands; however, a direct link between these two phenomena has been established only for HIV $[64,65]$.

In cancer cells, the first link between DDR and upregulation of NKG2D ligand expression was provided by Gasser and coworkers: they demonstrated that genotoxic stress conditions are responsible for persistent NKG2D ligand expression mainly through ATM, ATR, and Chk1 activation, thus increasing the sensitivity of tumor cells to NK cell-mediated lysis [33]. Studies from our laboratory demonstrated that genotoxic chemotherapy drugs enhance the expression of NKG2D ligands via induction of the DDR-dependent E2F1 transcription factor, and that this effect is preferentially associated with the induction of a senescence phenotype, supporting the conclusion that drug-induced senescence represents a mechanism that contributes to the elimination of tumor cells [34,61]. Notably, p53, which is also a downstream mediator of ATM/ATR pathways, is not essential for upregulation of murine NKG2DL expression [33], but can amplify the transcription of particular human NKG2D ligands $[66,67]$.

Additional stress pathways (e.g., the heat shock pathway, the oxidative stress pathway, and the endoplasmic reticulum stress response) are also involved in NKG2D ligand regulation, and may act cooperatively with the DDR pathway to ensure that cell-surface expression of NKG2D ligand is restricted to unhealthy cells $[2,3]$.
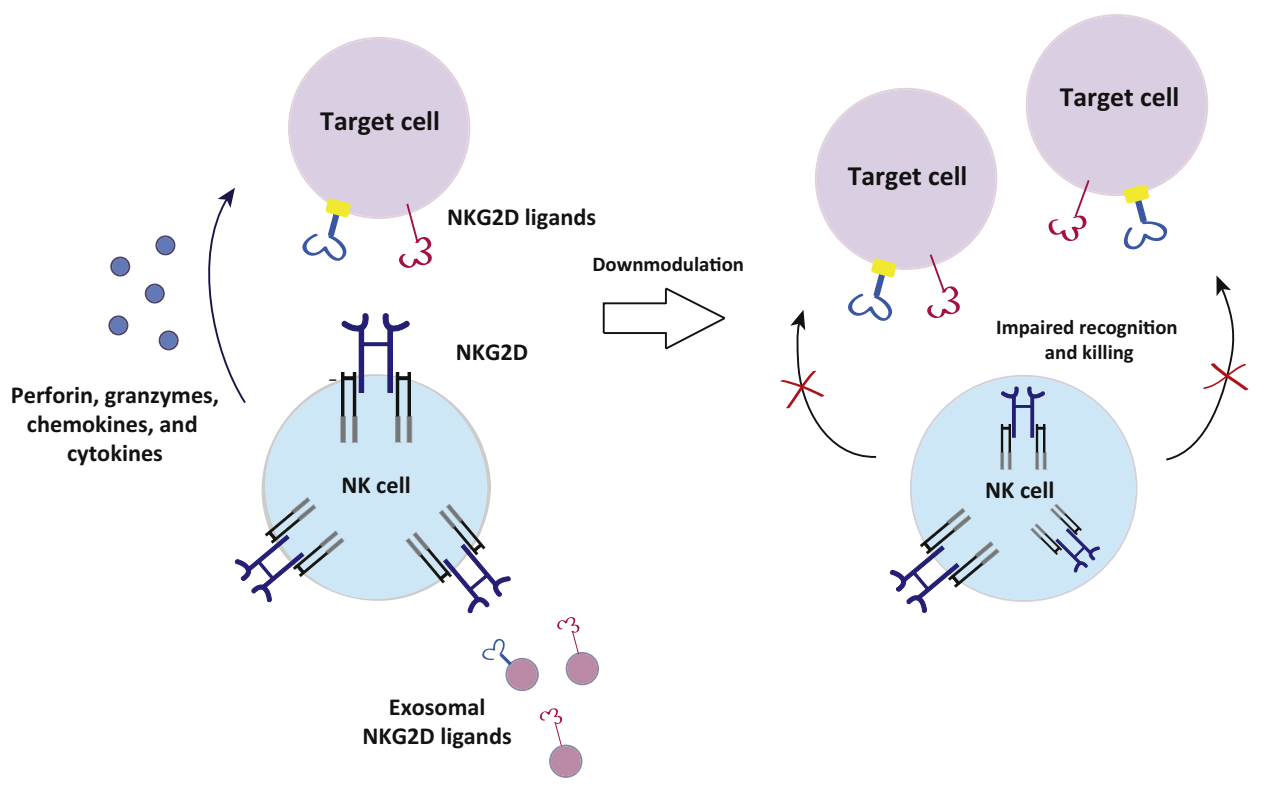

Trends in Immunology

Figure 2. Impact of NKG2D Downmodulation on Natural Killer (NK) Cell Functions. The expression of NKG2D ligands renders target cells susceptible to NK cell-mediated lysis. However, chronic exposure to membrane and exosomal NKG2D ligands promotes receptor downmodulation on human NK cells that renders them hyporesponsive to NKG2D ligand-bearing tumor cells. 
Table 1. NKG2D Ligands ${ }^{a}$

\begin{tabular}{|c|c|c|}
\hline Human NKG2D ligands & Cell-surface attachment ${ }^{b}$ & Affinity, $K_{d}(\mathrm{M})$ \\
\hline MICA & TM & $0.9-1 \times 10^{-6}$ \\
\hline MICB & TM & $8 \times 10^{-7}$ \\
\hline ULBP1 & GPI & $1.1 \times 10^{-6}$ \\
\hline ULBP2 & GPI & ND \\
\hline ULBP3 & GPI & ND \\
\hline ULBP4 & TM & ND \\
\hline ULBP5 & TM & ND \\
\hline ULBP6 & GPI & ND \\
\hline Mouse NKG2D ligands & Cell-surface attachment ${ }^{b}$ & Affinity, $K_{\mathrm{d}}(\mathrm{M})$ \\
\hline RAE1 $\propto$ & GPI & $7 \times 10^{-7}$ \\
\hline RAE1 $\beta$ & GPI & $3-19 \times 10^{-7}$ \\
\hline RAE1 $\gamma$ & GPI & $5-6 \times 10^{-7}$ \\
\hline RAE1 $\delta$ & GPI & $7-8 \times 10^{-7}$ \\
\hline RAE1 $\varepsilon$ & GPI & $3 \times 10^{-8}$ \\
\hline $\mathrm{H} 60 \mathrm{a}$ & TM & $2-3 \times 10^{-8}$ \\
\hline $\mathrm{H} 60 \mathrm{~b}$ & TM & $3 \times 10^{-7}$ \\
\hline $\mathrm{H} 60 \mathrm{c}$ & GPI & $9 \times 10^{-6}$ \\
\hline MULT1 & TM & $6 \times 10^{-9}$ \\
\hline
\end{tabular}

aData from [1,2].

${ }^{\mathrm{b}}$ Abbreviations: GPI, glycosylphosphatidylinositol; TM, transmembrane.

modes of membrane anchoring (TM and GPI-linked, respectively) and/or to differences in their affinity/avidity for NKG2D (Table 1).

The consequences of NKG2D ligand-receptor interaction have been further investigated in murine models. Localized receptor downmodulation leading to functional impairment of NK cells and dermal resident $\gamma \delta$ T cells was reported in an in vivo model of Rae-1 overexpression in normal epithelium [12]. In in vitro experiments, H60 membrane-bound ligand downregulated NKG2D expression and its associated functions [14]. Together, these results demonstrate that mouse membrane-bound ligands share with their human counterparts the ability to induce NKG2D downregulation, and that receptor downmodulation does not necessarily require a tumor context and can also be elicited by ligands expressed on healthy cells.

Unlike the case of cell-bound ligands, the ability of soluble ligands to downregulate their receptor and impair NKG2D-mediated cytolytic functions is still debated. Groh and coworkers showed that chronic stimulation of normal $\mathrm{CD}^{+} \mathrm{T}$ cells with sera containing soluble MICA/B derived from patients with different epithelial cancers efficiently downmodulated NKG2D expression and impaired receptor function in CD8 ${ }^{+}$T cells [10]. Moreover, the reduction of NKG2D expression found on NK cells derived from patients with colorectal cancer correlated with the presence of soluble MIC ligand in sera from patients, and rendered NK cells unable to lyse $\mathrm{MIC}^{+}$autologous tumor cells [48]. In contrast with these findings, only membrane-bound NKG2D ligands were shown to induce NKG2D downmodulation in multiple myeloma patients, even though soluble ligands were found in their sera [50]. In the context of autoimmune diseases, high concentrations of soluble MICA were found in the sera of patients; however, NKG2D surface expression was not affected [36,37]. 
Similarly, a functional impact of soluble NKG2D ligands released into culture supernatants is not always observed. ULBP2 released into the supernatants of transfected cells by metalloproteinase-mediated shedding did not affect NKG2D expression on NK cells [15], while high concentrations of recombinant soluble ULPB2 reduced NK cell activity by decreasing NKG2D surface expression [51]. A different situation has been observed in human activated T cells where $\mathrm{MICB}$ released in vitro from activated $\mathrm{CD}^{+}$lymphocyte is the major factor responsible for reduction of NKG2D expression on $\mathrm{CD} 8^{+} \mathrm{T}$ cells upon 5 days of $\mathrm{CD} 4^{+} / \mathrm{CD} 8^{+}$co-culture [52].

Notably, a recent study reported a novel scenario in which a soluble form of the highest-affinity murine ligand, MULT1, promotes tumor rejection [53]. Indeed, tumor cells engineered to secrete MULT1 were rejected when injected into syngeneic mice, likely via NK cell activation. Based on these findings, the authors hypothesized that chronic NKG2D engagement on NK cells by NKG2D ligands present on the cell surface of non-tumor cells leads to NKG2D downmodulation and general NK cell desensitization, whereas soluble MULT1 blocks these interactions and increases NK cell responsiveness. In the presence of soluble MULT1, NK cells were thus not desensitized, and were able to eliminate tumor cells, likely after recognition through other activating receptors. Thus, these results strongly argue against a role of soluble monomeric NKG2D ligands in immune evasion.

Some considerations related to the mechanisms underlying NKG2D ligand release could help to resolve these conflicting results. Soluble NKG2D ligands could be either shed after proteolytic cleavage or released in exosomes $[45,46]$, and these latter forms appear to be more potent downmodulators than their shed counterparts. In the case of MIC ligands, supernatants containing exosome-released $\mathrm{MICA}^{*} 008$ were shown to decrease NKG2D expression at the NK cell surface more potently than supernatant containing high concentrations of metalloproteinase-shed MICA [45]. Accordingly, Fernandez-Messina and coworkers demonstrated that exosome-released ULBP3 is better able to downmodulate receptor expression than the metalloproteinase-shed ULBP2 ligand [46]. This likely depends on the fact that multiple ULBP3 molecules are exposed at the exosome surface, thus engaging NKG2D with high avidity. Additional evidence also supports a role for exosomal NKG2D ligands in promoting receptor downmodulation. Chronic exposure to tumor-derived exosomes containing MIC ligands downmodulated human NKG2D surface expression on both NK and CD8 ${ }^{+} \mathrm{T}$ lymphocytes, and rendered them less prone to produce IFN- $\gamma$ and to kill tumor cells [49].

NKG2D downmodulation upon persistent exposure to NKG2D ligand-bearing exosomes has been also reported in other contexts. For example, during pregnancy the syncytiotrophoblast can release exosomes bearing NKG2D ligands that decrease NKG2D surface expression on maternal circulating lymphocytes, resulting in inhibition of the NKG2D-dependent cytotoxic response [54].

Concerning the possibility that NKG2D downregulation can cross-tolerize unrelated activating receptors, leading to a more general functional impairment of cell functions, conflicting results have been reported. Upon transient interaction (18 h) with MICA and ULBP2, human NK cells downmodulate NKG2D expression and function, but maintain cytotoxic potential triggered by other activating receptors [16]. However, longer in vitro stimulation with NKG2DLs might impact on functions unrelated to NKG2D. In fact, the functional capacity of CD3 $\zeta$-associated receptors was impaired in $\mathrm{CD}^{+} \mathrm{T}$ lymphocytes and NK cells upon 3 days of co-culture with MICAexpressing cells [55]. Reduced levels of $\mathrm{CD} 3 \zeta$ were also observed in murine NK cells stimulated for 3 days with H60-expressing targets [14], and H6O-experienced NK cells showed an impairment of NKG2D-dependent and- independent cytotoxicity [56]. These discrepancies can be explained considering that the functional outcome may vary depending on the length of NKG2D stimulation and/or on the type of ligand involved. In vivo, conflicting results were 


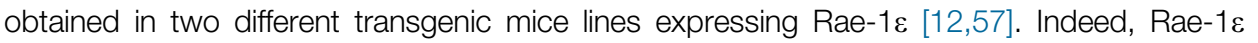
overexpression in the FVB mouse strain resulted in systemic NKG2D downregulation that provokes defects in NK cell cytotoxicity against MHC class I-deficient target cells [12]. However, transgenic mice expressing the same NKG2D ligand on the C57BL/6 background showed defects in NKG2D-dependent NK cell functions, but efficiently rejected MHC class I-deficient splenocytes [57]. These different results may depend on the employment of murine models that differ in Rae1 $\varepsilon$ expression levels and/or NK cell lytic activity.

Together, these lines of evidence demonstrate that both membrane-bound and exosomal NKG2D ligands efficiently downmodulate the NKG2D receptor and ultimately reduce the sensitivity of cytotoxic lymphocyte to target cells expressing NKG2D ligands. Persistent and chronic NKG2D stimulation may eventually cause a more general NK cell functional impairment.

\section{Molecular Mechanisms Regulating NKG2D Expression and Signaling: Role of Endocytosis}

Endocytosis is the main mechanism responsible for NKG2D downmodulation, although intercellular transfer (trogocytosis) of NKG2D to target cells via cytotoxic immune synapses has been proposed to explain, at least in part, the loss of receptor from the plasma membrane [58]. Upon ligand binding, both human and murine NKG2D internalization is inhibited by sucrose pretreatment [11,52], suggesting that receptors are mainly internalized by clathrin-mediated endocytosis [11,52]. However, the involvement of additional clathrin-independent endocytic pathways cannot be excluded and has not yet been explored.

Regarding the fate of the internalized receptor, we and others have demonstrated that, upon internalization, the human NKG2D/DAP10 receptor complex traffics through the endosomal compartment and is routed towards lysosomes where both NKG2D and DAP10 are rapidly degraded $[10,16,59]$. However, the rate of receptor internalization and degradation may depend on the nature of the ligand. Indeed, the $\mathbf{E} 3$ ubiquitin ligase $\mathrm{c}-\mathrm{Cbl}$ is required for MICA- but not ULBP2-induced NKG2D endocytosis, leading to more-rapid receptor lysosomal degradation [16].

Notably, upon MICA binding, human DAP10 itself undergoes mono- and poly-ubiquitination, and this modification provides a signal that directs internalization of NKG2D from the plasma membrane and its delivery to lysosomes [17] (Figure 3, Key Figure). The type of polyubiquitin chains (Lys48- or Lys63-linked) and the mechanism through which ubiquitinated NKG2D receptors are coupled to endocytic machinery remain to be established. It also remains unknown whether $\mathrm{c}-\mathrm{Cbl}$ is the ubiquitin ligase responsible for this modification and whether MICB also activates the ubiquitin pathway and promotes DAP10 ubiquitination.

In line with the results obtained with MICA, the expression of a fusion protein in which ubiquitin was fused to the C-terminal end of DAP10 in mouse resulted in constitutive NKG2D/DAP10 receptor-complex degradation [24], suggesting that the ubiquitin pathway is also involved in murine NKG2D endocytosis. However, conflicting results were obtained regarding the fate of internalized murine NKG2D. Three days of stimulation of murine NK cells with transfected cells expressing the NKG2D ligand H60 reduced the total protein amounts of both NKG2D-associated adapters (DAP10 and DAP12) [14], supporting a degradative receptor fate. By contrast, upon $24 \mathrm{~h}$ of interaction with Rae1 $\varepsilon$-bearing targets, NKG2D internalization was not followed by receptor degradation in NOD mice [11].

Together, these findings suggest that the fate of internalized murine receptor complexes depends on the type of NKG2D ligand. Moreover, even though receptor internalization appears 
Key Figure

Proposed Model of NKG2D/DAP10 Endocytosis and Signaling upon Ligand Binding

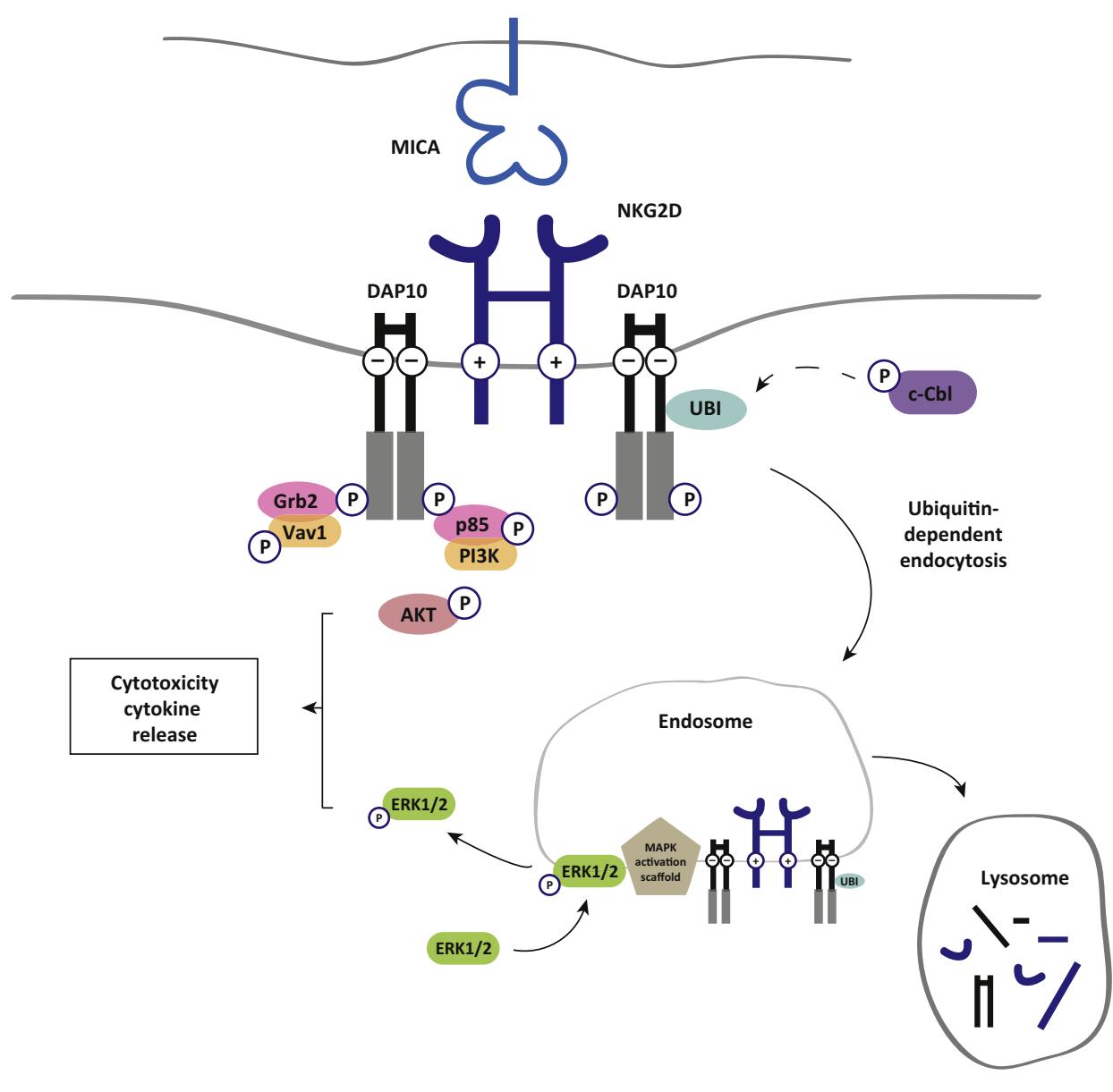

Trends in Immunology

Figure 3. In human NK cells, NKG2D engagement by MICA activates the ubiquitin ligase c-Cbl and promotes DAP10 ubiquitination that provides a signal for NKG2D internalization and lysosomal degradation. On the other hand, ubiquitindependent receptor internalization and traffic along endosomes is required for the phosphorylation of ERK $1 / 2$ on endosomal membrane platforms, before the receptors reach lysosomes and undergo degradation. Activated ERK $1 / 2$ proteins then detach from endosomes or translocate to the nucleus to reach their substrates, thus allowing signal propagation and NK cell effector functions. Figure modified from [17]. Abbreviations: DAP: DNAX-associated protein; ERK, extracellular signal-regulated kinase; MIC, MHC class I related protein; NK cell, natural killer cell; P, phosphorylation; PI3K, phosphoinositide-3 kinase; UBI, ubiquitin.

to be a rapid process, NKG2D degradation in mouse may require a longer period of stimulation than in human.

Recent findings also demonstrate that ubiquitin-dependent receptor endocytosis is indispensable for intracellular signal propagation, allowing NKG2D-mediated NK cell function [17]. In particular, proximal events such as Vav1 phosphorylation and PI3K activation are initiated at the plasma membrane and do not require ubiquitin-dependent receptor endocytosis, whereas the 


\section{Box 2. Endocytosis: Impact on Immune Receptor-Mediated Signaling}

The interplay between endocytosis and signaling has been initially documented from studies on ligand-induced downregulation of epidermal growth factor receptors (EGFRs) and other receptor tyrosine kinases (RTKs), as well as of G protein-coupled receptors (GPCRs) $[68,69]$. Upon ligand binding, receptor-mediated cellular signaling promotes receptor endocytosis through the recruitment of molecular sorting machineries that control cargo transport into endocytic compartments and ultimately to lysosomes for degradation. However, the rate of internalization of such receptors is substantially higher than the rate of their degradation. As a consequence, there is a prolonged residence of internalized receptors in endosomes, and these act as platforms where signaling is sustained. Moreover, changes in the localization of activated receptors (from the cell surface to endosomal compartments) can affect the composition of signaling complexes, thus regulating not only the extent but also the quality of cellular responsiveness [70].

With regard to activating receptors expressed on immune cells, several lines of evidence support the concept that endosomes can act to initiate and/or sustain receptor-mediated signal. This scenario is exemplified by the Toll-like receptors (TLR): TLR3, TLR7, and TLR9 are localized mostly on endosomes and require ligand internalization to initiate signaling [71], whereas TLR4 can activate diverse signaling pathways based on its location (plasma membrane versus endosomes), leading to the production of different inflammatory cytokines [72].

In the hematopoietic cytokine receptor context, IL-4R-mediated JAK/STAT signaling is guaranteed by the preferential concentration of ligand-induced receptor dimers within a specific population of endosomes associated with the cell cortex [73]. Similarly, KIR2DL4, an activating receptor expressed on NK cells and some T cell subsets, must accumulate in early endosomes to initiate a proinflammatory cascade $[74,75]$. In B cells, intracellular trafficking of the B cell receptor (BCR) controls the extent of MAPK activation and Akt signaling [76], whereas TCR internalization ensures the appropriate signaling strength that is required to promote the proliferation of mouse T cells in vivo [77].

With respect to NKG2D-DAP10 on human NK cells, as discussed in this review, the finding that receptor internalization is rapidly followed by receptor lysosomal degradation suggests that endosomal signaling serves to allow signal propagation rather than to sustain it. Moreover, in the context of NK cells, endosomes may represent platforms for the integration of signals triggered by different co-engaged receptors, thus adding an extensive and complex level of functional regulation.

full activation of extracellular signal-regulated kinases 1 and 2 (ERK1/2) occurs on endosomal compartments [17]. Indeed, a dramatic impairment of ERK1/2 phosphorylation was observed in NK cells in which ligand-induced NKG2D endocytosis was inhibited either by cell pretreatment with a selective inhibitor of dynamin GTPase activity or by dynamin-2 silencing. Moreover, both internalized receptor complexes and pERK1/2 transiently localize in early endosomes, supporting the conclusion that NKG2D internalization is required for ERK1/2 activation in endosomal compartments [17]. These results demonstrate that NKG2D endocytosis is instrumental for signal propagation and NK cell functional responses.

The interplay between endocytosis and signaling has been documented in different cell context and for various types of receptors, supporting the notion that endosomes can act as platforms to initiate and/or sustain receptor-mediated intracellular signals (Box 2). In the case of NKG2DDAP10 receptor complexes, internalization was rapidly followed by their lysosomal degradation, suggesting that the contribution of endosomal signaling would serve to guarantee the amplitude of MAPK/ERK signal propagation.

Together, these results suggest that the internalized NKG2D/DAP10 receptors contribute to the assembly of an endosomal scaffold complex to which ERK, a key signaling element in driving NK cell cytotoxicity [60], is recruited and phosphorylated. Phosphorylated ERK can then detach from endosomes to reach its targets, thus allowing signal propagation and functional responses (Figure 3).

\section{Concluding Remarks and Future Directions}

NKG2D ligand expression represents a danger signal that renders damaged cells susceptible to NK cell-mediated elimination. However, persistent contact of effector cells with NKG2DLexpressing targets causes receptor downmodulation, thus impairing NKG2D-mediated cell functions. Intriguingly, in human NK cells internalized NKG2D/DAP10 receptor complexes

\section{Outstanding Questions}

Are soluble ligands shed from tumor cells able to induce NKG2D downregulation? Although several lines of evidence support a role for exosomal ligands in NKG2D downregulation, conflicting results were reported for shed ligands.

Does NKG2D ubiquitination occur upon engagement with membrane-bound ligands other than MICA? The ubiquitin pathway and c-Cbl direct MICA- but not ULBP2-induced NKG2D internalization and lysosomal degradation. Whether MICB and/or ULPB ligands other than ULBP2 could activate the ubiquitin pathway and promote DAP10 ubiquitination is currently unknown.

Are different membrane-bound NKG2D ligands equivalently able to promote signaling from endosomes?

Is NKG2D endocytosis necessary to ensure appropriate signaling strength also in the context of T lymphocytes?

What is the impact of NKG2D endocytosis on other unrelated NK cell-activating receptor-dependent functions?

Could NK cell-activating receptors other than NKG2D promote signaling from endosomes?

The functional response of an NK cell depends on a delicate balance of signals from activating and inhibitory receptors, and thus compartmentalization of key signaling components between the plasma membrane and endosomes could control the outcome of pathways initiated upon receptor engagement. 
continue to signal in the endosomes until they are degraded, thereby ensuring full activation of key signaling components, such as ERK1/2, that are necessary for the functional outcome. Because the functional NK cell response is the result of the integration of signals derived from activating and inhibitory receptors, it is interesting to speculate that compartmentalization of transducing elements between cell-surface and endosomal membranes may preserve a sufficient amount of active receptor complexes in signaling-competent endocytic compartments to overcome inhibitory signals. A different scenario may be envisaged for activated murine NK cells, where ITAM-mediated signaling by NKG2D/DAP12 complexes may make the contribution of endosomal signal propagation dispensable.

A general open question is how endosome-based signaling is terminated. The ubiquitinmediated transfer of activated receptors into intraluminal vesicles, as well as receptor degradation in the lysosomal compartment, represent well-established mechanisms that could apply also for engaged NKG2D. Another possible mechanism is through endosome acidification, a process long recognized to promote ligand-receptor dissociation and modulate growth factor responses. However, whether endosomal signaling requires continued NKG2D engagement with its ligands in the endosome has not been addressed so far.

An additional open question will be to elucidate whether NKG2D endocytosis is also necessary to ensure the appropriate signaling strength in the context of T cell co-stimulation (see Outstanding Questions).

Furthermore, considering that human NKG2D ligands differ in their ability to induce receptor internalization and degradation, it would be interesting to further investigate whether distinct ligands could also differ in their ability to promote signaling from endosomes.

\section{Acknowledgments}

We apologize to all our colleagues whose important work could not be cited directly. Most of these references can be found in the review articles cited in the manuscript. Work in our laboratory is supported by grants from the Italian Association for Cancer Research (AIRC), the Italian Ministry for University and Research (MIUR-FIRB; PRIN/20103FMJEN/AngelaSantoni), and the Center of Excellence (BEMM). We thank Dr John Hiscott (Director of Research, the Institute Pasteur Laboratory, Rome, Italy) for critical reading of the manuscript.

\section{References}

1. Eagle, R.A. and Trowsdale, J. (2007) Promiscuity and the single receptor: NKG2D. Nat. Rev. Immunol. 7, 737-744

2. Raulet, D.H. et al. (2013) Regulation of ligands for the NKG2D activating receptor. Annu. Rev. Immunol. 31, 413-441

3. Ullrich, E. et al. (2013) New prospects on the NKG2D/NKG2DL system for oncology. Oncoimmunology 2, e26097

4. Lanier, L.L. (2015) NKG2D receptor and its ligands in host defense. Cancer Immunol. Res. 3, 575-582

5. Cerwenka, A. et al. (2001) Ectopic expression of retinoic acid early inducible-1 gene (RAE-1) permits natural killer cell-mediated rejection of a MHC class I-bearing tumor in vivo. Proc. Natl. Acad. Sci. U. S. A. $98,11521-11526$

6. Diefenbach, A. et al. (2001) Rae1 and H60 ligands of the NKG2D receptor stimulate tumour immunity. Nature 413, 165-171

7. Smyth, M.J. et al. (2005) NKG2D function protects the host from tumor initiation. J. Exp. Med. 5, 583-588

8. Guerra, N. et al. (2008) NKG2D-deficient mice are defective in tumor surveillance in models of spontaneous malignancy. Immunity $28,571-580$

9. Zafirova, B. et al. (2009) Altered NK cell development and enhanced NK cell-mediated resistance to mouse cytomegalovirus in NKG2D-deficient mice. Immunity 31, 270-282

10. Groh, V. et al. (2002) Tumour-derived soluble MIC ligands impair expression of NKG2D and T-cell activation. Nature 419, $734-738$
11. Ogasawara, K. et al. (2003) Impairment of NK cell function by NKG2D modulation in NOD mice. Immunity 18, 41-51

12. Oppenheim, D.E. et al. (2005) Sustained localized expression of ligand for the activating NKG2D receptor impairs natural cytotoxicity in vivo and reduces tumor immunosurveillance. Nat. Immunol. 6, 928-937

13. Wiemann, K. et al. (2005) Systemic NKG2D down-regulation impairs NK and CD8 T cell responses in vivo. J. Immunol. 175 720-729

14. Coudert, J.D. et al. (2005) Altered NKG2D function in NK cells induced by chronic exposure to NKG2D ligand-expressing tumor cells. Blood 106, 1711-1717

15. Waldhauer, I. and Steinle, A. (2006) Proteolytic release of soluble UL16-binding protein 2 from tumor cells. Cancer Res. 66, 25202526

16. Molfetta, R. et al. (2014) c-Cbl regulates MICA- but not ULBP2 induced NKG2D down-modulation in human NK cells. Eur. J. Immunol. 44, 2761-2770

17. Quatrini, L. et al. (2015) Ubiquitin-dependent endocytosis of NKG2D-DAP10 receptor complexes activates signaling and func tions in human NK cells. Sci. Signal. 8, ra108

18. Garrity, D. et al. (2005) The activating NKG2D receptor assembles in the membrane with two signaling dimers into a hexameric structure. Proc. Natl. Acad. Sci. U. S. A. 102 $7641-7646$ 
19. Bauer, S. et al. (1999) Activation of NK cells and T cells by NKG2D, a receptor for stress-inducible MICA. Science 285, 727-729

20. Wu, J. et al. (1999) An activating immunoreceptor complex formed by NKG2D and DAP10. Science 285, 730-732

21. Diefenbach, A. et al. (2002) Selective associations with signaling proteins determine stimulatory versus costimulatory activity of NKG2D. Nat. Immunol. 3, 1142-1149

22. Gilfillan, S. et al. (2002) NKG2D recruits two distinct adapters to trigger NK cell activation and costimulation. Nat. Immunol. 3, $1150-1155$

23. Billadeau, D.D. et al. (2003) NKG2D-DAP10 triggers human NK cell-mediated killing via a Syk-independent regulatory pathway. Nat. Immunol. 4, 557-564

24. Horng, T. et al. (2007) NKG2D signaling is coupled to the interleukin 15 receptor signaling pathway. Nat. Immunol. 8, 1345-1352

25. Graham, D.B. et al. (2006) Vav1 controls DAP10-mediated natural cytotoxicity by regulating actin and microtubule dynamics. J. Immunol. 177, 2349-2355

26. Upshaw, J.L. et al. (2006) NKG2D-mediated signaling requires a DAP10-bound Grb2-Vav1 intermediate and phosphatidylinositol3-kinase in human natural killer cells. Nat. Immunol. 7. 524-532

27. Bryceson, Y.T. et al. (2009) Minimal requirement for induction of natural cytotoxicity and intersection of activation signals by inhibitory receptors. Blood 114, 2657-2666

28. Ohashi, M. et al. (2010) Post-translational modification of the NKG2D ligand RAET1G leads to cell surface expression of a glycosylphosphatidylinositol-linked isoform. J. Biol. Chem. 285, 16408-16415

29. Fernández-Messina, L. et al. (2011) The human NKG2D ligand ULBP2 can be expressed at the cell surface with or without a GPI anchor and both forms can activate NK cells. J. Cell Sci. 124, 321-327

30. Ashiru, O. et al. (2013) A GPI anchor explains the unique biological features of the common NKG2D-ligand allele MICA*008. Biochem. J. 454, 295-302

31. Groh, V. et al. (1996) Cell stress-regulated human major histocompatibility complex class I gene expressed in gastrointestinal epithelium. Proc. Natl. Acad. Sci. U. S. A. 93, 12445-21250

32. Cosman, D. et al. (2001) ULBPs, novel MHC class I-related molecules, bind to CMV glycoprotein UL16 and stimulate NK cytotoxicity through the NKG2D receptor. Immunity 14, 123-133

33. Gasser, S. et al. (2005) The DNA damage pathway regulates innate immune system ligands of the NKG2D receptor. Nature 436, 1186-1190

34. Soriani, A. et al. (2009) ATM-ATR-dependent up-regulation of DNAM-1 and NKG2D ligands on multiple myeloma cells by therapeutic agents results in enhanced NK-cell susceptibility and is associated with a senescent phenotype. Blood 113, 3503-3511

35. Cerboni, C. et al. (2014) The DNA damage response: a common pathway in the regulation of NKG2D and DNAM-1 kigand expression in normal, infected, and cancer cells. Front Immunol. 4, 508

36. Groh, V. et al. (2003) Stimulation of T cell autoreactivity by anomalous expression of NKG2D and its MIC ligands in rheumatoid arthritis. Proc. Nat. Acad. Sci. U. S. A. 100, 9452-9457

37. Hüe, S. et al. (2004) A direct role for NKG2D/MICA interaction in villous atrophy during celiac disease. Immunity 21, 367-377

38. Meresse, B. et al. (2004) Coordinated induction by IL15 of a TCR-independent NKG2D signaling pathway converts CTL into lymphokine-activated killer cells in celiac disease. Immunity 21, 357-366

39. Champsaur, M. and Lanier, L.L. (2010) Effect of NKG2D ligand expression on host immune responses. Immunol. Rev. 235, 267-285

40. Bacon, L. et al. (2004) Two human ULBP/RAET1 molecules with transmembrane regions are ligands for NKG2D. J. Immunol. 173, 1078-1084

41. Cao, W. et al. (2007) RAET1E2, a soluble isoform of the UL16binding protein RAET1E produced by tumor cells, inhibits NKG2Dmediated NK cytotoxicity. J. Biol. Chem. 282, 18922-18928

42. Salih, H.R. et al. (2002) Cutting edge: down-regulation of MICA on human tumors by proteolytic shedding. J. Immunol. 169, 4098-4102
43. Boutet, P. et al (2009) Cutting edge: the metalloproteinase ADAM17/TNF-alpha-converting enzyme regulates proteolytic shedding of the MHC class I-related chain B protein. J. Immunol. $182,49-53$

44. Zingoni, A. et al. (2015) Genotoxic stress induces senescenceassociated ADAM10-dependent release of NKG2D MIC ligands in multiple myeloma cells. J. Immunol. 195, 736-748

45. Ashiru, O. et al. (2010) Natural killer cell cytotoxicity is suppressed by exposure to the human NKG2D ligand MICA*008 that is shed by tumor cells in exosomes. Cancer Res. 70, 481-489

46. Fernández-Messina, L. et al. (2010) Differential mechanisms of shedding of the glycosylphosphatidylinositol (GPI)-anchored NKG2D ligands. J. Biol. Chem. 285, 8543-8551

47. Park, Y.P. et al. (2011) Complex regulation of human NKG2DDAP10 cell surface expression: opposing roles of the $\gamma_{c}$ cytokines and TGF- $\beta 1$. Blood 118, 3019-3027

48. Doubrovina, E.S. et al. (2003) Evasion from NK cell immunity by $\mathrm{MHC}$ class I chain-related molecules expressing colon adenocarcinoma. J. Immunol. 171, 6891-6899

49. Clayton, A. et al. (2008) Human tumor-derived exosomes downmodulate NKG2D expression. J. Immunol. 180, 7249-7258

50. von Lilienfeld-Toal, M. et al. (2010) Reduced immune effector cell NKG2D expression and increased levels of soluble NKG2D ligands in multiple myeloma may not be causally linked. Cancer Immunol. Immunother. 59, 829-839

51. Song, H. et al. (2006) Soluble ULBP suppresses natural killer cell activity via down-regulating NKG2D expression. Cell. Immunol. 239, 22-30

52. Cerboni, C. et al. (2009) Detuning $C D 8^{+} T$ lymphocytes by down-regulation of the activating receptor NKG2D: role of NKG2D ligands released by activated T cells. Blood 113, 2955-2964

53. Deng, W. et al. (2015) A shed NKG2D ligand that promotes natural killer cell activation and tumor rejection. Science 348 , 136-139

54. Hedlund, M. et al. (2009) Human placenta expresses and secretes NKG2D ligands via exosomes that down-modulate the cognate receptor expression: evidence for immunosuppressive function. $J$. Immunol. 183, 340-351

55. Hanaoka, N. et al. (2010) NKG2D initiates caspase-mediated CD3zeta degradation and lymphocyte receptor impairments associated with human cancer and autoimmune disease. $J$. Immunol. 185, 5732-5742

56. Coudert, J.D. et al. (2008) Sustained NKG2D engagement induces cross-tolerance of multiple distinct NK cell activation pathways. Blood 111, 3571-3578

57. Champsaur, M. et al. (2010) Intact NKG2D-independent function of NK cells chronically stimulated with the NKG2D ligand Rae-1. J. Immunol. 185, 157-165

58. Roda-Navarro, P. et al. (2006) Transfer of NKG2D and MICB at the cytotoxic NK cell immune synapse correlates with a reduction in NK cell cytotoxic function. Proc. Natl. Acad. Sci. U. S. A. 103, 11258-11263

59. Roda-Navarro, P. and Reyburn, H.T. (2009) The traffic of the NKG2D/Dap10 receptor complex during natural killer (NK) cell activation. J. Biol. Chem. 284, 16463-16472

60. Galandrini, R. et al. (2013) Activation of Iymphocyte cytolytic machinery: where are we? Front. Immunol. 4, 390

61. Soriani, A. et al. (2014) Reactive oxygen species- and DNA damage response-dependent NK cell activating ligand upregulation occurs at transcriptional levels and requires the transcriptional factor E2F1. J. Immunol. 193, 950-960

62. Cerboni, C. et al. (2007) Antigen-activated human T lymphocytes express cell-surface NKG2D ligands via an ATM/ATR-dependent mechanism and become susceptible to autologous NK-cell lysis. Blood 110, 606-615

63. Jung, H. et al. (2012) RAE-1 ligands for the NKG2D receptor are regulated by E2F transcription factors, which control cell cycle entry. J. Exp. Med. 209, 2409-2422

64. Ward, J. et al. (2009) HIV-1 Vpr triggers natural killer cell-mediated lysis of infected cells through activation of the ATR-mediated DNA damage response. PLoS Pathog. 5, e1000613 
65. Richard, J. et al. (2010) HIV-1 Vpr up-regulates expression of ligands for the activating NKG2D receptor and promotes NK cell-mediated killing. Blood 115, 1354-1363

66. Li, H. et al. (2011) Pharmacological activation of p53 triggers anticancer innate immune response through induction of ULBP2. Cell Cycle 10, 3346-3358

67. Textor, S. et al. (2011) Human NK cells are alerted to induction of p53 in cancer cells by upregulation of the NKG2D ligands ULBP1 and ULBP2. Cancer Res. 71, 5998-6009

68. Irannejad, R. et al. (2015) Effects of endocytosis on receptormediated signaling. Curr. Opin. Cell Biol. 35, 137-143

69. Bergeron, J.J. et al. (2016) Spatial and temporal regulation of receptor tyrosine kinase activation and intracellular signal transduction. Annu. Rev. Biochem. 85, 573-597

70. Murphy, J.E. et al. (2009) Endosomes: a legitimate platform for the signaling train. Proc. Natl. Acad. Sci. U. S. A. 106, 17615-17622

71. Brubaker, S.W. et al. (2015) Innate immune pattern recognition: a cell biological perspective. Annu. Rev. Immunol. 33, 257-290
72. Kagan, J.C. et al. (2008) TRAM couples endocytosis of Toll-like receptor 4 to the induction of interferon-beta. Nat. Immunol. 9 , 361-368

73. Kurgonaite, K. et al. (2015) Essential role of endocytosis for interleukin-4-receptor-mediated JAK/STAT signalling. J. Cell Sci. 128 3781-3795

74. Rajagopalan, S. et al. (2006) Activation of NK cells by an endocytosed receptor for soluble HLA-G. PLoS Biol. 4, es

75. Miah, S.M. et al. (2011) Ubiquitylation of an internalized killer cell Ig like receptor by Triad3A disrupts sustained NF-KB signaling. $J$. Immunol. 186, 2959-2969

76. Chaturvedi, A. et al. (2011) Endocytosed BCRs sequentially regulate MAPK and Akt signaling pathways from intracellular compartments. Nat. Immunol. 12, 1119-1126

77. Willinger, T. et al. (2015) Dynamin 2-dependent endocytosis sustains $T$-cell receptor signaling and drives metabolic reprogramming in T lymphocytes. Proc. Natl. Acad. Sci. U. S. A. 112 4423-4428 